\title{
Synthetic Matrices to Serve as Niches for Muscle Cell Transplantation
}

\author{
Sarah Fernandes ${ }^{a-c}$ Shannon Kuklok ${ }^{\mathrm{e}}$ Joe McGonigle ${ }^{\mathrm{e}}$ Hans Reinecke ${ }^{\mathrm{a}-\mathrm{c}}$ \\ Charles E. Murry ${ }^{\mathrm{a}-\mathrm{d}}$ \\ ${ }^{a}$ Center for Cardiovascular Biology, ${ }^{b}$ Institute for Stem Cell and Regenerative Medicine, and Departments of \\ 'Pathology and ${ }^{\mathrm{d} B i o e n g i n e e r i n g ~ a n d ~ M e d i c i n e / C a r d i o l o g y, ~ U n i v e r s i t y ~ o f ~ W a s h i n g t o n, ~ S e a t t l e, ~ W a s h ., ~ a n d ~}$ \\ esurModics, Inc., Eden Prairie, Minn., USA
}

\section{Key Words}

Biomaterial $\cdot$ Heart · Cellular therapy

\begin{abstract}
Poor cell retention and limited cell survival after grafting are major limitations of cell therapy. Recent studies showed that the use of matrices as vehicles at the time of cell injection can significantly improve cell engraftment by providing an appropriate structure and physical support for the injected cells. Properly designed matrices can also promote the organization of the cells into a functioning cardiac-like tissue and enhance integration between the host and the engrafted tissue. Furthermore, the use of an injectable biomaterial provides an opportunity to release in situ bioactive molecules that can further enhance the beneficial effects of cell transplantation. In this article we review a large variety of biologically derived synthetic and hybrid materials that have been tested as matrices for cardiac repair. We summarize the optimal parameters required for an ideal matrix including biocompatibility, injectability, degradation rate, and mechanical properties. Using an in vivo subcutaneous grafting model, we also provide novel data involving a side-by-side comparison of six synthetic matrices derived from maltodextrin. By systematically varying polymer molecular weight, cross-link density, and availability of cell adhesion motifs, a synthetic matrix was identified that supported skeletal myo-
\end{abstract}

tube formation similar to Matrigel ${ }^{\mathrm{TM}}$. Our results emphasize not only the need to have a range of tunable matrices for cardiac cell therapy but also the importance of further characterizing the physical properties required for an ideal injectable matrix.

Copyright $\odot 2011$ S. Karger AG, Base

\section{KARGER}

Fax +4161306 1234

E-Mail karger@karger.ch

www.karger.com
(C) 2011 S. Karger AG, Basel

1422-6405/12/1952-0048\$38.00/0

Accessible online at:

www.karger.com/cto

\section{Abbreviations used in this paper}

DE

DMEM

DPBS

ESCs

GFP

$\mathrm{H} / \mathrm{E}$

$\mathrm{Hi} / \mathrm{Hep}$

$\mathrm{Hi} /$ Cross

matrix

Hi/Hi matrix

dextrose equivalent

Dulbecco's modified Eagle's medium

Dulbecco's phosphate-buffered saline

embryonic stem cells

green fluorescent protein

hematoxylin/eosin

matrix containing an alternative electrophilic polymer composition that incorporates a heparin moiety $\mathrm{Hi} / \mathrm{Hi}$ polymer matrix with an increased level of cross-linking

matrix formed with two high molecular weight polymers

Hi/HiNoRGD Hi/Hi polymer matrix without RGD-containing matrix peptide

Hi/Lo matrix matrix formed with high and low molecular weight polymers

IGF1 insulin-like growth factor 1

Lo/Lo matrix matrix formed with high and low molecular weight polymers

PAS periodic acid Schiff

RGD peptide arginine-glycine-aspartic acid 


\section{Introduction}

Cardiomyocytes lost after myocardial infarction are replaced by noncontractile scar tissue, leading to decreased myocardial function, negative cardiac remodeling, and progression toward heart failure. Traditional pharmacological treatments focus on diminishing the workload and improving the systolic performance of the heart (e.g. beta blockers, diuretics, and vasodilators). While this strategy can slow disease progression, ultimately heart transplantation remains the only treatment option for end-stage heart failure. Unfortunately, the need for donor hearts vastly exceeds the supply, making heart transplantation an option for relatively few patients. In this context, cell transplantation offers a promising alternative strategy for patients with acute and chronic heart failure. The predominant goal of cardiac cell therapy is to remuscularize and revascularize the damaged myocardium and, thereby, to restore cardiac function of the infarcted heart. Early studies focused largely on committed myogenic cell sources such as skeletal myoblasts [Murry et al., 1996; Jain et al., 2001; Pouzet et al., 2001; Leobon et al., 2003] or fetal/neonatal cardiomyocytes [Leor et al., 1996; Scorsin et al., 2000]. More recently, the beneficial effects of bone marrow-derived cells [Scorsin et al., 2000; Kocher et al., 2001; Nygren et al., 2004; Forest et al., 2010], resident cardiac progenitor cells [Tang et al., 2010], and derivatives of embryonic stem cells (ESCs) [Caspi et al., 2007; Laflamme et al., 2007a; Leor et al., 2007] have also been evaluated.

Although cell therapy for cardiac repair has shown promising results in preclinical studies and some clinical trials [Murry et al., 2006; Laflamme et al., 2007b, 2011], poor retention and/or survival of the transplanted cells in the infarcted area remains a major limitation [Robey and Murry, 2008; Anderl et al., 2009]. When direct intramyocardial injections are performed, greater than $50 \%$ of cells escape from the heart within $1 \mathrm{~h}$ due to leakage from the needle track or migration into coronary veins after injection [Yasuda et al., 2005a, b; Anderl et al., 2009]. Of the remaining cells, only $15 \%$ and less than $5 \%$ are present at 1 and 6 weeks, respectively [Zhang et al., 2001; Müller-Ehmsen et al., 2002]. While the immediate cell loss after intramyocardial injection is mainly caused by leakage from the injection site, a major cause of cell loss over the subsequent weeks is so-called anoikis: a programmed death initiated by lack of cell attachment and matrix support [Robey et al., 2008; Anderl et al., 2009]. Therefore, the success of regenerative cell therapy for cardiac repair will initially require a means of increasing the number of surviving cells at the treatment site.

Synthetic Matrices for Cell Transplantation
Injectable extracellular matrices have been studied for cell delivery vehicles to maximize retention and survival of cells. The first part of this article reviews the principal requirements of and the recent advances in the search for the ideal injectable matrix for cardiac cell therapy. In the second part, we provide preliminary results regarding the ability of a novel type of maltodextrin-derived hydrogel to support cell survival and differentiation of myogenic cells in vivo using a high-throughput subcutaneous grafting model in the mouse.

\section{Part 1: Review of the Literature}

\section{Requirements of an Ideal Injectable Matrix for Cell Therapy in Cardiac Repair}

The primary goals for the use of an injectable matrix are to improve graft size by enhancing cell retention and survival and to promote integration of the muscular, vascular, and connective tissue compartments between the graft and the host. These goals are complicated by the fact that the heart is a solid organ with continuous contraction-relaxation cycles. The high metabolic rate of heart muscle requires dense vascularization, and the tissue organization is complex, with overlapping layers of muscle arranged in different circumferential orientations. Therefore, the task of a good matrix to integrate is, in fact, very complex. Minimal requirements of the ideal matrix include: (1) biocompatibility (i.e. nonimmunogenic), (2) mechanical properties similar to those of the heart tissue, (3) allowance of biological exchange between the engrafted cells and the host tissue, (4) facilitation of structural and functional integration between newly formed and existing tissue, and (5) gradual degradation (without toxic/immunogenic byproducts) as the implanted cells secrete their own matrix [Bell, 1995].

For practical reasons, the matrices need to remain liquid until after their injection and then solidify quickly after delivery in vivo. In this context, thermoplastic (i.e. temperature-dependent) gelling and cross-linked (triggered by mixing two separate components just before injection) gelling have been studied. The time to gel needs to be adapted for each delivery method, e.g. a short time period for direct intramyocardial injection during thoracotomy or a longer time period when catheter-based delivery is desired.

Because of their early commercial availability, most research to date focused on matrices of biological origin or derivatives thereof. Examples include fibrin glue, collagen foam, gelatin, alginate, chitosan, and Matrigel ${ }^{\mathrm{TM}}$

Cells Tissues Organs 2012;195:48-59 
matrix. More recently, biomimetic polymers and synthetic hydrogels modified with short amino acid sequences to reproduce the native extracellular matrix chemistry have been synthesized and evaluated using in vitro and in vivo models. Properties of synthetic matrices can be built in by design, e.g. with the desired porosity, mechanical stability, and degradation properties. Importantly they can be synthesized in a reproducible, controlled process, thus offering large-scale production and minimizing the risk of disease transmission or impurities [Wall et al., 2010]. Despite our best efforts, some degree of cell death after implantation appears unavoidable. Since cell degradation products are toxic to other cells, the matrix should allow for host macrophages to infiltrate in order to remove cellular debris. In addition, depending on the cell type injected, beneficial cytokine production by the transplanted cells may occur [Nelson et al., 2011]. Thus, the matrix should allow for diffusion of these cytokines in order to exert maximal effects on the graft cells (autocrine mechanism) and the host tissue (paracrine mechanism). Similarly, the matrix should allow for the transport of nutrients and oxygen influx and support host vessel in-growth, i.e. neovascularization, of the engrafted region. Encapsulation fibrosis has been seen in xenotransplantation and allotransplantation [van Laake et al., 2010] with or without matrix coinjection. This is of particular importance for cardiac cell therapy where the presence of electrically insulated islands can disrupt the normal conduction of the electrical signal within the heart, thus leading to life-threatening arrhythmia. For this reason, a good matrix should facilitate the electromechanical coupling between the injected cardiomyocytes and the host myocardium.

\section{In vivo Studies}

Because the heart is a mechanically active solid organ, an adequate matrix should provide appropriate stress transfer during loading and unloading cycles. Several groups have been evaluating injectable matrices in an acellular approach to support the injured left ventricular wall and to prevent adverse remodeling. Injection of Matrigel [Kofidis et al., 2005], alginate [Kofidis et al., 2005], fibrin glue [Christman et al., 2004a], and collagen [Dai et al., 2005] alone into infarcted heart muscle was thought to decrease wall stress in the remaining myocardium, to reduce left ventricular dilation, and to increase angiogenesis. Some relatively stiff matrices have been shown to have beneficial effects on the contractile function of the heart. However, a side-by-side comparison showed that fibrin (less stiff) is superior to alginate (more stiff) in reducing the final infarct scar size [Yu et al., 2009]. Mechanical properties of matrices also provide cues with regard to proliferation, differentiation, and phenotype for numerous cell types including adult mesenchymal stem cells [Jacot et al., 2010], skeletal myoblasts [Gilbert et al., 2010], and cardiomyocytes [Jacot et al., 2008; Bajaj et al., 2010]. For example, optimal sarcomeric organization in cardiomyocytes (determined by actinmyosin cross-striations) was observed on a substrate mimicking the stiffness of the myocardium, indicating the importance of the matrix mechanical properties on cell maturation [Engler et al., 2008; Jacot et al., 2008; Bhana et al., 2010].

To date, numerous studies have demonstrated that the beneficial effect of intramyocardial cell injection for cardiac repair is enhanced by the use of injectable matrices. In rodent models of myocardial infarction, Matrigel can potentiate the beneficial effect of ECSs and their derivatives, e.g. cardiomyocytes [Kofidis et al., 2005; Laflamme et al., 2007a]. In a similar study, Kraehenbuel et al. [2011] showed that the injection of a bioactive hydrogel alone can improve contractile function and decrease adverse remodeling of the rat heart, but again this effect was potentiated with hESC-derived vascular cells.

Matrices may also be beneficial in reducing ectopic relocation of the injected cells. For example, it was shown that mesenchymal stem cells delivered in a collagen matrix remained in the heart, whereas cell delivery in saline solution resulted in ectopic relocation to other organs [Dai and Kloner, 2010]. In contrast, using an acutely ischemic rat heart model, Anderl et al. [2009] demonstrated that although injectable matrices can reduce immediate leakage they do not appear to effect retention measured 20 min after microsphere injection. Similarly, Christman et al. [2004b] showed that fibrin glue or saline vehicle has similar effects on myoblast retention at $24 \mathrm{~h}$ after intramyocardial injection. However, at a 4 -week time point, graft size was significantly bigger in the fibrin glue group, suggesting an impact of the extracellular matrix on the proliferation of engrafted cells.

A variety of materials can accelerate spontaneous in situ vascularization for example by promoting endothelial progenitor cell mobilization, adhesion, and proliferation. The interactions between cell surface receptors and extracellular matrix molecules have been described [for a review see de Mel et al., 2008]. Indeed, when injected in vivo most of the tested matrices have been shown to improve vascularization. To accelerate angiogenesis and engraftment, the implanted scaffold can be impregnated with bioactive molecules [Leor et al., 2005]. The targeted 
and controlled delivery of pharmacologic reagents to the local environment may improve cell transplantation efficiency not only by increasing the neovascularization but also by attenuating the inflammatory response, improving the viability and survival of the injected cells and enhancing stem cell homing and self-repair. The injection of hESC-derived vascular cells embedded in a synthetic bioactive hydrogel together with the proangiogenic and prosurvival factor thymosin $\beta 4$ enhanced contractile performance in a rat model of myocardial infarction compared to the hydrogel plus cells alone [Kraehenbuehl et al., 2011]. Similarly, Davis et al. [2006] designed an injectable peptide that self-assembles into randomly branched networks. The resulting nanofiber microenvironment was shown to support long-term controlled release of the cytokine insulin-like growth factor 1 (IGF1) in a rat infarction model resulting in an improvement in systolic function [Davis et al., 2006]. Administration of cardiac progenitor cells together with self-assembling nanofibers capable of releasing IGF1 augmented the expression of connexin 43 and $\mathrm{N}$-cadherin in newly formed cardiomyocytes to foster electrical and mechanical coupling between resident and regenerated myocytes [PadinIruegas et al., 2009]. The importance of such electrical integration is demonstrated by the increased incidence of ventricular arrhythmias when skeletal myoblasts are engrafted into the myocardium. Skeletal myoblasts do not express detectable levels of connexin 43 in vivo [Reinecke et al., 2000; Fernandes et al., 2006] and thus have a low capacity to transmit electrical signals.

While most studies reported potentiation of cell therapy when matrices are used as a vehicle, some studies have described nonbeneficial effects [Dubois et al., 2008; Dai et al., 2009; Dai and Kloner, 2010]. For example, Dubois et al. [2008] reported deleterious effects of one formulation of self-assembling peptide nanofibers on cell survival in vitro. Thus, cell/matrix/tissue compatibility can never be assumed and must be tested for each donor cell and host tissue combination.

\section{Part 2: A Novel Synthetic Matrix Based on Modified Maltodextrins}

Several lessons emerge from the aforementioned studies. Most importantly, matrices that can significantly enhance cell engraftment may potentiate the therapeutic effect of cell transplantation. In order to obtain optimal benefits, it is important that the characteristics of any given candidate matrix are adapted to the cell type and the target tissue, e.g. the infarcted heart. However, testing many different matrices in the background of an infarct model is a labor- and cost-intensive and inefficient process. Many years of experience with in vivo grafting have taught us that high interanimal variability is intrinsic to all cardiac grafting models. In addition, cardiomyocyte preparations obtained from ESCs or from neonatal heart preparations are heterogeneous and do not guarantee consistent cardiomyocyte content. On the other hand, in vitro experiments do not reliably predict the outcomes of complex in vivo injury models. We aimed to design an in vivo study that offers high throughput at a low cost while minimizing interanimal variability. Therefore, we chose a muscle cell line (C2C12 skeletal myoblasts) and a subcutaneous grafting model. Importantly, a previous study from our laboratory [Robey et al., 2008] showed that C2C12 survival correlates with human ESC-derived cardiomyocyte survival and long-term engraftment in the intramyocardial injection model in the mouse. Therefore, for the initial evaluation we decided to utilize this myogenic immortalized cell line in a subcutaneous injection model in immunocompromised mice. Using this model we were able to screen several matrix conditions in the same animal while the use of the $\mathrm{C} 2 \mathrm{C} 12$ cell line ensured minimal variability with regard to the cell preparations, thus emphasizing the matrices as the unique variable between groups. The potential of several novel in situ cross-linked matrices based on maltodextrin was evaluated based on the survival and differentiation/maturation of the injected cells.

\section{Materials and Methods}

\section{Description of in situ Cross-Linked Matrices}

The experimental matrices consist of a two-component system which cross-links upon mixing to form a hydrogel. These matrices have several properties that make them well suited for cell therapy applications. First, they are based on a maltodextrin-derived polymer backbone, which is highly water soluble and predicted to biodegrade in vivo by the action of ubiquitous tissue amylases. Furthermore, the properties of the matrix are tunable through changing the polydispersity of the polymer backbone, the degree of functional group modification, and the concentrations of the two polymers. Changing these properties is expected to modulate important parameters for matrix performance including gel time and physicomechanical properties of resultant gels. Finally, this polymer system consists entirely of synthetic or plant-derived material which reduces concerns of using matrices containing animal-derived material such as collagen or Matrigel matrix.

The primary components in this system are polymers consisting of maltodextrin derivatized with functional groups. Maltodextrin is a saccharide polymer that consists of D-glucose units 
linked primarily by (alpha)-1-4 bonds and has a dextrose equivalent (DE) of less than 20 [Bender, 2005]. DE is a measure of the degree of hydrolysis of starch into dextrose and reflects the total percentage of solids converted into reducing sugars such that dextrose has a DE of 100 and starch has a DE of 0 . For these studies, the maltodextrin backbone was separately modified with one of two functional groups to produce two different component polymers - a nucleophile and an electrophile - intended to undergo a nucleophilic substitution reaction with one another at neutral $\mathrm{pH}$ to spontaneously form the hydrogel. At neutral $\mathrm{pH}$, these functional groups are expected to a have greater affinity for each other as opposed to various functional groups found on cells. The cells therefore are expected to be mechanically incorporated in the matrix without covalent modification.

The two polymers are dissolved in Dulbecco's phosphate-buffered saline (DPBS) and the nucleophile-derivatized polymer component is combined with cells. The nucleophile polymer/cell solution is then mixed with the second electrophile-derivatized polymer solution, ultimately yielding a cross-linked hydrogel containing entrapped cells. In all matrices but one, the nucleophile polymer component was also further modified with a short arginine-glycine-aspartic acid (RGD)-containing peptide sequence intended to promote cell attachment to the matrix.

In order to screen for a potential matrix for eventual cardiac cell therapy, we used a mouse model with subcutaneous injection of myogenic cells. Skeletal myoblast (mouse C2C12 line) viability, differentiation, and maturation were evaluated with six separate matrix formulations at three different time points (3 days, 2 weeks, and 1 month). In all matrices but one, the nucleophilic polymer was modified with a short RGD-containing peptide sequence intended to promote cell attachment to the matrix. Three formulations systematically compared the effect of varying molecular weight characteristics of the polymer backbone. One matrix was formed by a combination of two low molecular weight polymers (Lo/Lo), another was a mixture of high and low molecular weight polymers (Hi/Lo), and the third matrix was a combination of two high molecular weight polymers (Hi/Hi). Two variations of the $\mathrm{Hi} / \mathrm{Hi}$ matrix were also evaluated, i.e. one where the theoretical level of cross-linking in the matrix was increased (Hi/Cross) and a second where the RGD-containing peptide in the nucleophilic polymer was omitted (Hi/HiNoRGD). The final matrix contained an alternative electrophilic polymer composition that incorporates a heparin moiety ( $\mathrm{Hi} / \mathrm{Hep})$. Twenty-four hours before injections, each polymer component was reconstituted with DPBS, sterile filtered $(0.22 \mu \mathrm{m})$, and kept at $4{ }^{\circ} \mathrm{C}$. Matrigel matrix (Growth Factor Reduced BD Matrigel ${ }^{\mathrm{TM}}$, BD Biosciences) was used as a benchmark control as it is known to promote cell survival and retention in vivo.

\section{Cell Preparation}

A mouse myoblast cell line (C2C12) stably transduced with green fluorescent protein (GFP) was used as a model stem cell line. C2C12 myoblasts were expanded in Dulbecco's modified Eagle's medium (DMEM) supplemented with L-glutamine, 20\% fetal bovine serum, and $1 \%$ penicillin/streptomycin. On the day of injection, cells were harvested by trypsinization. After counting, cells were washed twice with DPBS containing glucose and sodium pyruvate, aliquoted, and resuspended in the adequate volume of nucleophile polymer. To evaluate clearance of cell debris, retention kinetics, and matrix degradation, dead cell preparations were obtained by performing three freeze-thaw cycles of the pelleted cells. Matrigel (BD Biosciences) was used as a benchmark control matrix. Once resuspended in polymer or Matrigel the cell suspensions (dead and live cells) were kept on ice until use.

\section{Subcutaneous Cell Injections}

An immunodeficient mouse model (SCID-beige spontaneous mutant model CBSCBG-MT; Taconic Farms, Inc.) was used to ensure engraftment of EGFP-expressing cells. This double mutant mouse carries the SCID mutation which causes a lack of both $\mathrm{T}$ and $\mathrm{B}$ lymphocytes, as well as the beige mutation which causes macrophage defects and impairs NK cell function [Schechner et al., 2000]. Mice (8-12 weeks) were anesthetized with isoflurane (2\%). Each mouse underwent six injections placed subcutaneously on the back. To minimize variability due to animal- or sitespecific effects, each mouse was injected with all six experimental matrices, and the location of the matrices was rotated for each mouse. Dead cell injections and live cell injections were not mixed in the same animal. Similarly, Matrigel injections were performed in separate animals. In total three injections were performed for each matrix condition (each matrix with both live and dead cells).

For all matrices and for all time points, cells from a given cell batch were injected on the same day by the same blinded researcher. For the experimental matrices, the electrophile polymer solution was added to the nucleophile polymer cell suspension just prior to injection. The final suspension was mixed, and $80 \mu \mathrm{l}$ containing $4 \times 10^{6}$ cells was quickly injected using an insulin syringe with a 29-gauge needle. The cell density was chosen to match that routinely used for intracardiac cell injections in mice commonly performed in our laboratory [Robey et al., 2008]. To minimize leakage of the injected cell-matrix preparation, the syringe was held in place for $20 \mathrm{~s}$ after cell injection.

\section{Histology}

In order to evaluate cell retention and differentiation kinetics, grafts were harvested at 3 days, 2 weeks, and 1 month after cell injection. Briefly, animals were euthanized by pentobarbital injection, and the back skin was removed. Visible grafts were harvested, cut in half to allow fixation with both methyl Carnoy's and zinc fixative solution, and processed routinely for histology. To ensure that all C2C12-GFP+ grafts were harvested, mice were observed under fluorescent illumination using a stereomicroscope (Nikon SMZ).

Tissue sections $(5 \mu \mathrm{m})$ were cut from paraffin-embedded specimens and stained with hematoxylin/eosin $(\mathrm{H} / \mathrm{E})$, periodic acid Schiff (PAS), or picrosirius red/fast green. In addition, slides were immunostained for desmin or CD31 as markers for muscle cells and endothelial cells, respectively. Stained slides were scanned using the NanoZoomer Digital Pathology System (Hamamatsu), digitized, and analyzed by a blinded operator.

\section{Results}

Gross examination did not reveal any differences between the matrices or between the dead cell and live cell grafts. When found, matrices were easily distinguishable from the host tissue by their disk- or sphere-like shape 
and pale, off-white color. Matrices were attached to the subcutaneous tissue layers but easily harvested. Matrix recovery was higher for experimental matrices containing live cells. At the 1-month time point, $61 \%$ of matrices containing live cells were recovered compared to only $25 \%$ for matrices containing dead cells. For Matrigel matrix controls, 2 out of 3 injections were recovered with live cells compared to only 1 out of 3 with dead cell injections.

\section{Histologic Evaluation: Dead Cell Injections}

Three days after subcutaneous injection, close examination of $\mathrm{H} / \mathrm{E}$ staining and desmin immunocytochemistry of matrices recovered from the dead cell group showed necrotic cell debris interspersed within an amorphous matrix. The maltodextrin matrix was readily identified by PAS staining. Some desmin-positive debris was found in 2 out of 5 matrices (formulation $\mathrm{Hi} / \mathrm{Lo}$ and $\mathrm{Hi} / \mathrm{Cross}$ ) along with some fragmented nuclei, suggesting slower cell clearance kinetics with these two formulations. Microscopic examination of the injected scaffolds recovered 1 month after injection confirmed our macroscopic evaluation that not all maltodextrin-derived and Matrigel matrices were degraded completely at 1 month after subcutaneous injection.

\section{Histologic Evaluation: Live Cell Injections}

When live cells were injected we observed the presence of live graft-derived cells with all matrix conditions at the 1 -month time point. This demonstrates that, once injected in vivo in a subcutaneous mouse model, $\mathrm{C} 2 \mathrm{C} 12$ cells can engraft and survive for up to 1 month with any of the evaluated maltodextrin-derived matrices. Because cell survival was observed at up to 1 month with all maltodextrin-derived matrix formulations, we compared the effect of maltodextrin-derived matrices on $\mathrm{C} 2 \mathrm{C} 12$ differentiation and myotube organization focusing on the 1-month time point. $\mathrm{C} 2 \mathrm{C} 12$ differentiation, myotube formation, fibrosis, and cell density were evaluated and compared to Matrigel injection, our 'gold standard' (fig. 1, 2).

When injected with Matrigel matrix, C2C12 myoblasts differentiate into multinucleated and striated myotubes (fig. 1). The engrafted area is surrounded by a fibrous capsule separating the graft from the host tissue. Myotubes are distributed evenly and collagen deposition within the graft is low (as shown by picrosirius red staining; fig. 2). However, the cell density within the graft appeared low. The amorphous, eosinophilic matrix surrounding the myotubes indicated that not all Matrigel is resorbed at 1 month and that extracellular matrix was preventing close apposition of myotubes.

Synthetic Matrices for Cell Transplantation
When injected with maltodextrin-derived matrices, cell distribution within the graft is more heterogeneous than in Matrigel-embedded cells (fig. 2). In the same graft, we can observe areas of high cell density and low cell density. Similarly, desmin immunostaining revealed patches of organized, multinucleated myotubes, with adjacent areas containing more isolated myotubes separated by either scaffold or newly synthesized extracellular matrix. Because, Matrigel and maltodextrin-derived matrix injections were performed at the same time, were delivered to the same animals, used the same cell batch and the same resuspension protocol, and were performed by the same researcher, it appears likely that the observed heterogeneity in cell distribution results from the maltodextrin-derived matrix properties rather than methodological variance.

Mature myotube formation varied considerably, depending on the maltodextrin matrix formulation. Myotube formation was poor with Hi/HiNoRGD, where desmin staining revealed few multinucleated myotubes within the graft and no cross-striations (fig. 1). Most of the desmin-positive cells remained as isolated small mononuclear cells surrounded by extracellular matrix. Importantly, cellular debris was observed within the graft, suggesting ongoing cell death. The $\mathrm{Hi} / \mathrm{Hi}$ and $\mathrm{Hi} / \mathrm{Cross}$ matrices were similar to the Hi/HiNoRGD matrix. At the 1-month time point most of the desmin-positive cells in $\mathrm{Hi} / \mathrm{Hi}$ and $\mathrm{Hi} / \mathrm{Cross}$ showed few elongated, multinucleated myotubes and instead showed irregular cell morphology. When present, multinucleated myotubes were in localized areas rather than distributed evenly throughout the matrices. Cellular debris was also observed in the $\mathrm{Hi} /$ $\mathrm{Hi}$ and $\mathrm{Hi} /$ Cross matrices, suggesting ongoing cell death.

Matrices Lo/Lo and Hi/Hep both showed greater myotube formation at the 1-month time point compared to the $\mathrm{Hi} / \mathrm{HiNoRGD}, \mathrm{Hi} / \mathrm{Hi}$, and $\mathrm{Hi} / \mathrm{Cross}$ matrices, and the desmin-positive cells were more uniformly distributed. No clear-cut sarcomeric cross-striations were observed in either Lo/Lo or Hi/Hep matrices. In matrix Lo/Lo there were fewer myotubes and more mononucleated myoblasts than in $\mathrm{Hi} / \mathrm{Hep}$. The single Hi/Hep sample recovered exhibited large multinucleated cell bodies rather than elongated myotubes, with enlarged nuclei compared to the cell size. On the other hand, elongated and multinucleated myotubes were observed with matrix $\mathrm{Hi} / \mathrm{Hep}$ at the 2 -week time point (in 2 replicates), suggesting that the lack of elongated myotubes at 4 weeks may be a sectioning artifact. However, at both 2 weeks and 1 month we observed DNA fragmentation in the Hi/Hep matrix, again suggesting that cell death was occurring.

Cells Tissues Organs 2012;195:48-59 
Fig. 1. Myoblast maturation and differentiation. Representative desmin immunostaining of the implants recovered at the 1-month time point. Cross-striations (insets) were observed only when myoblasts were associated with Matrigel matrix or the maltodextrin-derived matrix resulting from the combination of high and low molecular weight polymers (Hi/Lo). Scale bar $=50 \mu \mathrm{m}$.
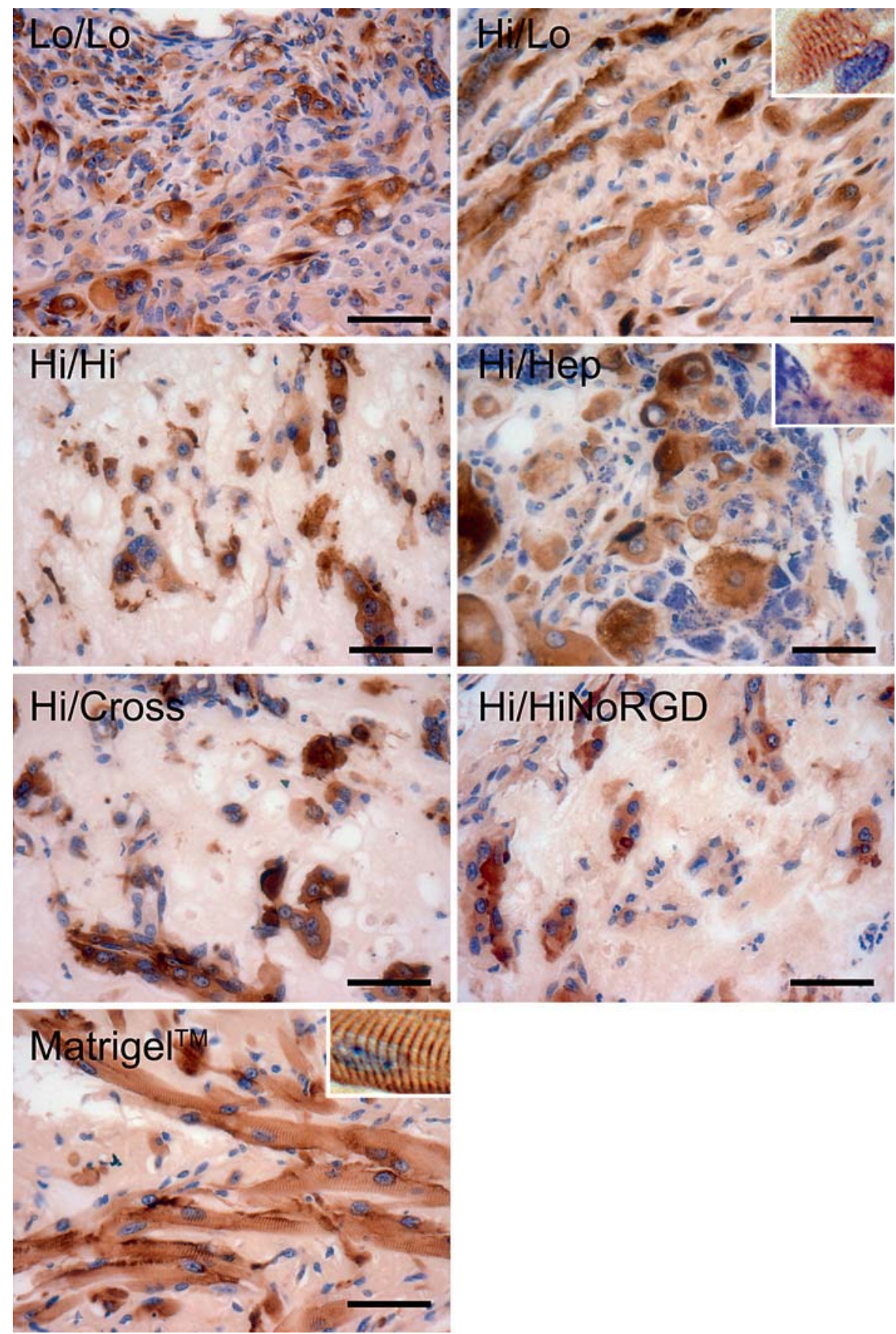

At the 1-month time point, 2 out of the 3 replicates with matrix Hi/Lo were recovered. In both specimens we observed the presence of abundant, elongated, multinucleated myotubes (fig. 3). Desmin staining demonstrated that these myotubes had readily identifiable sarcomeres similar to those observed in Matrigel grafts. Interestingly, the myotubes were evenly distributed throughout the graft and showed some degree of cell alignment. In Matrigel and Hi/Lo grafts, many of the nuclei of striated myotubes were positioned at the periphery of the cells, providing evidence for higher maturation in these two scaffolds. There was no obvious nuclear fragmentation, suggesting the absence of ongoing cell death. In matrix $\mathrm{Hi} / \mathrm{Lo}$, however, picrosirius red staining revealed a high 
Fig. 2. Collagen deposition depends on the matrix. At the 1-month time point, H/E (left panels) and picrosirius red (right panels) staining showed that each matrix is associated with variable levels of collagen deposition. The lowest level of collagen is observed with Matrigel, whereas the highest collagen level corresponds to the matrix combining high and low molecular weight monomers (Hi/Lo). Scale bar $=50 \mu \mathrm{m}$.
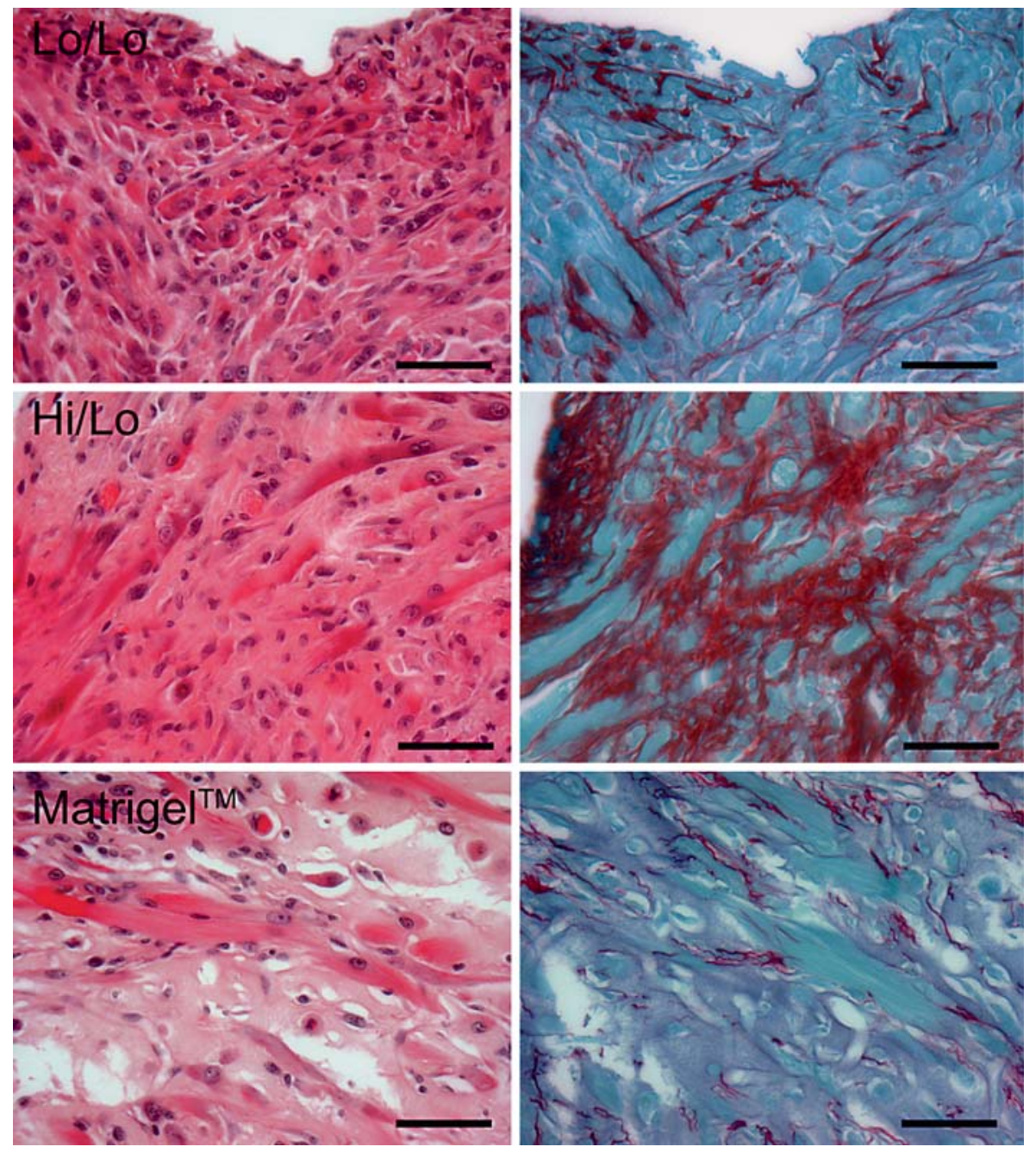

level of collagen within the extracellular matrix of the graft (fig. 2); it was significantly higher than that observed with Matrigel. In contrast, collagen was basically absent in the $\mathrm{Hi} / \mathrm{Hi}, \mathrm{Hi} / \mathrm{Cross}$, and $\mathrm{Hi} / \mathrm{HiNoRGD}$ formulations and very low in the Lo/Lo and No/Hep condition.

PAS (detecting carbohydrate moieties) was performed in order to evaluate the presence of the maltodextrin-derived matrices within the engrafted area. At the 3-day time point, PAS staining revealed abundant presence of matrices around the cells (fig. 3). PAS staining performed at the 1-month time point revealed presence of the maltodextrin-derived matrices under all matrix conditions although, interestingly, much of the PAS-positive material was within macrophages (fig. 3d). This indicates that the biodegradation of the matrices was still not complete by 4 weeks and that macrophages play an important role in the clearance of the material. In $\mathrm{Hi} / \mathrm{Hi}$ and $\mathrm{Hi} /$ Cross ex- periments, we observed large patches of extracellular maltodextrin scaffold surrounding islands of $\mathrm{C} 2 \mathrm{C} 12$ cells. In Lo/Lo and Hi/HiNoRGD conditions, residual maltodextrin matrices were detected mostly within macrophages, indicating ongoing biodegradation. There was minimal residual maltodextrin matrix in the Hi/Lo and $\mathrm{Hi} / \mathrm{Hep}$ conditions.

To evaluate vascularization of the graft, CD31 immunostaining was performed in a subset of zinc-fixed samples at the 3-day and 1-month time points. No vessel formation was observed at the 3-day time point (fig. 3). At 1 month after engraftment, we observed vascularization of the grafted area in matrices Lo/Lo, $\mathrm{Hi} / \mathrm{Lo}, \mathrm{Hi} / \mathrm{Hi}$, and $\mathrm{Hi} /$ Cross. In those conditions CD31 showed clear capillary formation within the grafts. Conversely, a lack of vessel formation was observed with the $\mathrm{Hi} / \mathrm{Hep}$ and $\mathrm{Hi} /$ HiNoRGD matrix conditions. 
Fig. 3. Hi/Lo matrix at the 3-day and 1-month time points. At the 3-day time point $(\mathbf{a}, \mathbf{c}, \mathbf{e}, \mathbf{g}), \mathrm{H} / \mathrm{E}$ (a) and PAS staining (c) showed extensive maltodextrin-derived matrix within the graft. At this same time point desmin immunostaining demonstrated the presence of mononuclear C2C12 myoblasts (e), and CD31 (g) was expressed in host tissue only but not in the graft area. At the 1-month time point $(\mathbf{b}, \mathbf{d}$, $\mathbf{f}, \mathbf{h}), \mathrm{H} / \mathrm{E}$ staining revealed the presence of multinucleated myotubes (b; arrows) and capillary lumens (b; inset). The matrix was still present (d; arrow) but we observed ongoing biodegradation of the matrix with presence of the matrix within macrophages (d; inset). Immunostaining for desmin and CD31 confirmed C2C12 differentiation into multinucleated myotubes (f) and graft vascularization by CD31+ endothelial cells (h). Scale bar $=100 \mu \mathrm{m}$.
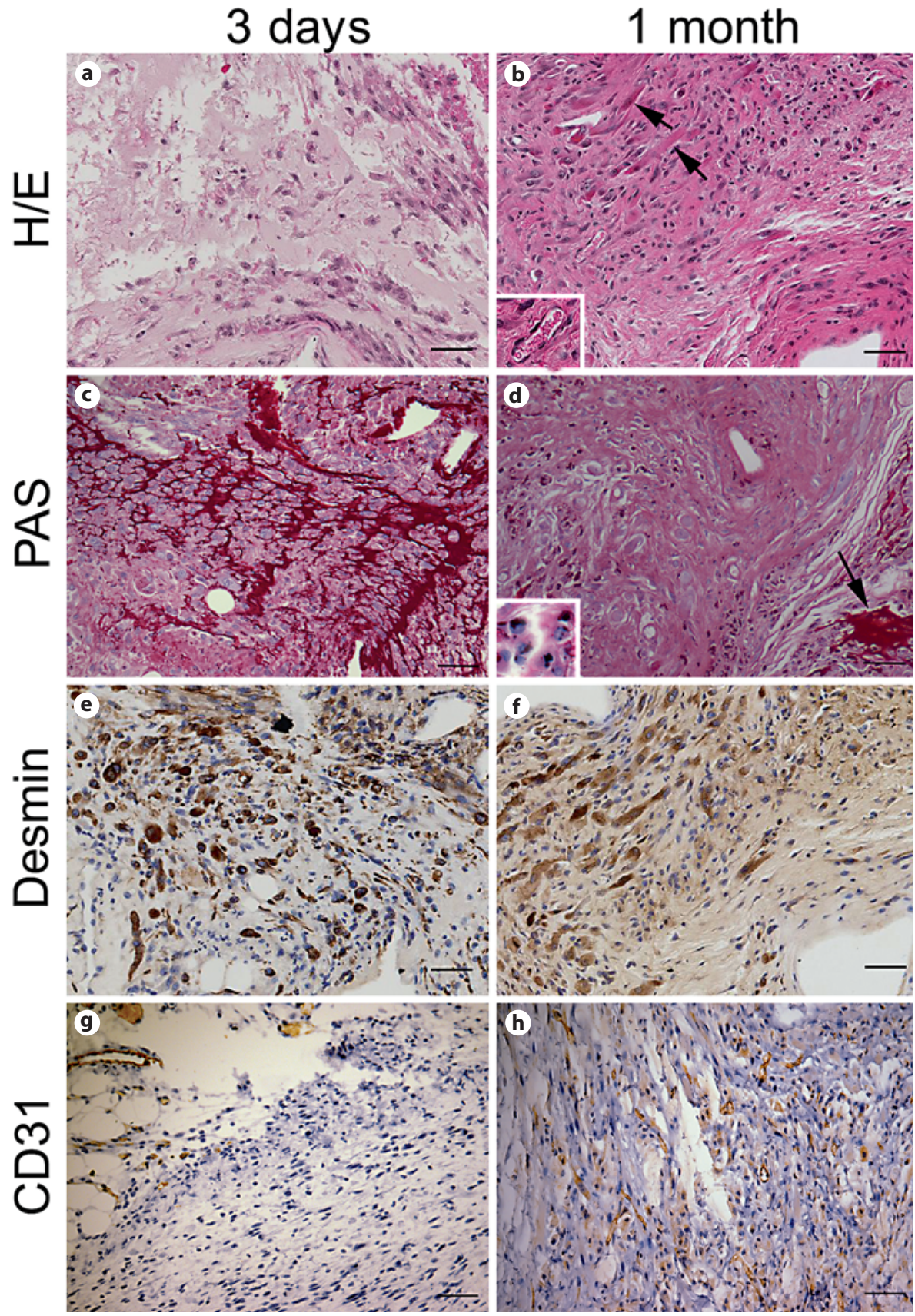

\section{Discussion}

The ability of a matrix to encourage myoblast differentiation and maturation is likely influenced by multiple bioengineering parameters, e.g. nanoarchitecture, porosity, and substrate compliance. All matrices evaluated by subcutaneous implantation study promoted retention of viable myoblasts up to 1 month after grafting in vivo.
However, we observed clear differences in myotube maturation (as evaluated by the presence of multinucleated myotubes and/or cross-striations) between the different maltodextrin-based matrix formulations. In fact, only one out of the six maltodextrin-based matrices (matrix $\mathrm{Hi} / \mathrm{Lo}$ ) showed a maturation level similar to our benchmark Matrigel. 
Several studies suggest that substrate stiffness may be a key parameter affecting myoblast proliferation and maturation [Engler et al., 2004; Boonen et al., 2009; Levy-Mishali et al., 2009]. Indeed, in vitro studies demonstrated that optimal myoblast differentiation occurred on substrates with a stiffness grade similar to that of muscle tissue. Interestingly, only a narrow window of stiffness grades allowed for the development of cross-striations, a sign of maturation, in vitro [Boonen et al., 2009]. In our study, matrices formed with the combination of two high molecular weight polymers (Hi/Hi, Hi/Cross, $\mathrm{Hi} / \mathrm{HiNoRGD})$ were characterized by low cell density within the graft, rare presence of multinucleated myotubes, and complete absence of cross-striations suggesting that these three matrices were too stiff to favor myoblast fusion and/or subsequent muscle cell maturation. Additionally, the RGD motif has been shown to promote the formation of the contractile apparatus in cultured myoblasts [LaNasa and Bryant, 2009]. Its removal from the maltodextrin-derived matrix in the Hi/HiNoRGD formulation may have augmented the adverse stiffness effect on myotube maturation.

Interestingly, the matrix Lo/Lo, resulting from the combination of two low molecular weight elements leading to a lower stiffness grade, also failed to support maturation as indicated by the lack of cross-striations. In this particular case, cell density within the graft appeared to be sufficient to permit cell fusion; nevertheless, the injected cells remained mononuclear. The lack of myotube formation has been observed previously in several studies where myoblasts were grown in vitro under low stiffness substrate conditions [Engler et al., 2004; Boonen et al., 2009; Levy-Mishali et al., 2009]. Interestingly, the Hi/ Hep formulation showed deformed clusters of myoblasts with enlarged nuclei consistent with a recent report of myoblast differentiation in a low stiffness matrix [LevyMishali et al., 2009]. In addition, low stiffness matrix conditions not only inhibit the development of the contractile apparatus in myotubes but also fail to provide proper adhesion-mediated signals necessary to induce the fusion and maturation pathways [García et al., 1999].

In our experiments, myotube formation and crossstriations indicating the development of the contractile apparatus were observed only with the $\mathrm{Hi} / \mathrm{Lo}$ formulation at the 1-month time point. These data suggest that only this particular matrix provided the adequate conditions required for myoblast fusion and subsequent maturation. In addition, striated myotube formation was accompanied by abundant collagen expression within the extracellular matrix. After muscle injury, muscle repair requires the production of extracellular matrix by fibro-

Synthetic Matrices for Cell

Transplantation blasts. Fibronectin and type III collagen are first synthetized, and then type I collagen is activated and remains elevated for several weeks, increasing the tensile strength of the scar [Kääriäinen et al., 2000]. The ECM components are then degraded as regeneration and growth of the new myofibers proceeds [Serrano and Muñoz-Cánoves, 2010]. A perturbation of any of these stages (insufficient ECM production at the early stage, excessive or persistent accumulation of ECM protein at the late stage) leads to impaired muscle regeneration. In our study, the best myotube formation (matrix Hi/Lo) was also the condition where most of the maltodextrin was degraded. This suggests that a more rapid degradation of the scaffold may promote myotube formation. Interestingly, we did not observe high levels of collagen deposition with the Matrigel condition. This might be explained by the fact that Matrigel itself provides enough support for myoblast differentiation/maturation. Because fibrosis can impair the integration of the newly injected cells with the host, $\mathrm{Hi} /$ Lo matrices would need to be evaluated at a later time point to assess collagen degradation.

Neovascularization of implanted matrices was observed in all conditions except for Hi/HiNoRGD and Hi/ Hep. The lack of vascularization in the Hi/HiNoRGD matrix as compared to the $\mathrm{Hi} / \mathrm{Hi}$ matrix indicates that the RGD moiety promotes vascularization after subcutaneous injection. RGD motifs are known to induce proangiogenic signaling in addition to serving as substrates for endothelial proliferation and migration [Moon and West, 2008]. The lack of vascularization in the Hi/Hep matrix, despite the presence of RGD, is potentially related to the cell death and fragmentation observed in this matrix. After injury, skeletal muscle satellite cells become activated and participate in endothelial cell signaling in order to facilitate revascularization in concert with skeletal muscle repair [Cornelison, 2008]. The secretion of cytokines like VEGF [Cornelison, 2008] or HIFlalpha [Ono et al., 2006] by the injected $\mathrm{C} 2 \mathrm{C} 12$ might favor vessel formation within the engrafted areas. The absence of significant vessel formation in the Hi/HiNoRGD and Hi/Hep matrices may be due to the lack of biological cues from the matrices themselves and/or to the absence of the appropriate cytokine secretion and cell signaling by the engrafted myoblasts.

\section{Conclusion}

In this study we provide a head-to-head comparison of several matrices to support myogenic cell survival and differentiation after subcutaneous in vivo implantation. 
Although all six matrices principally supported cell survival at 1 month after implantation (albeit at various degrees), we noticed clear differences with regard to myotube formation and maturation. By systematically varying polymer length, cross-link density, and the availability of cell adhesion motifs, we identified a formulation that performed comparably in relation to our benchmark matrix, Matrigel (commonly used in cell therapy). This points to the importance of tunability in designing a synthetic matrix for cell therapy. Maltodextrin scaffolds may prove useful for cell therapy applications. However, because cell-matrix interactions need to be evaluated for each graft cell type/target tissue combination, further studies are needed to determine their suitability for delivering cells to the heart for cardiac repair.

\section{References}

Anderl, J.N., T.E. Robey, P.S. Stayton, C.E. Murry (2009) Retention and biodistribution of microspheres injected into ischemic myocardium. J Biomed Mater Res A 88: 704-710.

Bajaj, P., X. Tang, T.A. Saif, R. Bashir (2010) Stiffness of the substrate influences the phenotype of embryonic chicken cardiac myocytes. J Biomed Mater Res A 95: 1261-1269.

Bell, E. (1995) Strategy for the selection of scaffolds for tissue engineering. Tissue Eng 1: 163-179.

Bender, D. (2005) A Dictionary of Food and Nutrition. New York, Oxford University Press.

Bhana, B., R.K. Iyer, W.L. Chen, R. Zhao, K.L. Sider, M. Likhitpanichkul, C.A. Simmons, M. Radisic (2010) Influence of substrate stiffness on the phenotype of heart cells. Biotechnol Bioeng 105: 1148-1160.

Boonen, K.J., K.Y. Rosaria-Chak, F.P. Baaijens, D.W. van der Schaft, M.J. Post (2009) Essential environmental cues from the satellite cell niche: optimizing proliferation and differentiation. Am J Physiol Cell Physiol 296: C1338-1345.

Caspi, O., I. Huber, I. Kehat, M. Habib, G. Arbel, A. Gepstein, L. Yankelson, D. Aronson, R. Beyar, L. Gepstein (2007) Transplantation of human embryonic stem cell-derived cardiomyocytes improves myocardial performance in infarcted rat hearts. J Am Coll Cardiol 50: 1884-1893.

-Christman, K.L., H.H. Fok, R.E. Sievers, Q. Fang, R.J. Lee (2004a) Fibrin glue alone and skeletal myoblasts in a fibrin scaffold preserve cardiac function after myocardial infarction. Tissue Eng 10: 403-409.

-Christman, K.L., A.J. Vardanian, Q. Fang, R.E. Sievers, H.H. Fok, R.J. Lee (2004b) Injectable fibrin scaffold improves cell transplant survival, reduces infarct expansion, and induces neovasculature formation in ischemic myocardium. J Am Coll Cardiol 44: 654-660.

Cornelison, D.D. (2008) Context matters: in vivo and in vitro influences on muscle satellite cell activity. J Cell Biochem 105: 663-669.

Dai, W., S.L. Hale, G.L. Kay, A.J. Jyrala, R.A Kloner (2009) Delivering stem cells to the heart in a collagen matrix reduces relocation of cells to other organs as assessed by nanoparticle technology. Regen Med 4: $387-$ 395.
Dai, W., R.A. Kloner (2010) Experimental cell transplantation therapy in rat myocardial infarction model including nude rat preparation. Methods Mol Biol 660: 99-109.

Dai, W., L.E. Wold, J.S. Dow, R.A. Kloner (2005) Thickening of the infarcted wall by collagen injection improves left ventricular function in rats: a novel approach to preserve cardiac function after myocardial infarction. J Am Coll Cardiol 46: 714-719.

Davis, M.E., P.C. Hsieh, T. Takahashi, Q. Song, S. Zhang, R.D. Kamm, A.J. Grodzinsky, P. Anversa, R.T. Lee (2006) Local myocardial insulin-like growth factor 1 (IGF-1) delivery with biotinylated peptide nanofibers improves cell therapy for myocardial infarction. Proc Natl Acad Sci USA 103: 8155-8160.

de Mel, A., G. Jell, M.M. Stevens, A.M. Seifalian (2008) Biofunctionalization of biomaterials for accelerated in situ endothelialization: a review. Biomacromolecules 9: 2969-2979.

-Dubois, G., V.F. Segers, V. Bellamy, L. Sabbah, S. Peyrard, P. Bruneval, A.A. Hagège, R.T. Lee, P. Menasché (2008) Self-assembling peptide nanofibers and skeletal myoblast transplantation in infarcted myocardium. J Biomed Mater Res B Appl Biomater 87: 222-228.

Engler, A.J., C. Carag-Krieger, C.P. Johnson, M. Raab, H.Y. Tang, D.W. Speicher, J.W. Sanger, J.M. Sanger, D.E. Discher (2008) Embryonic cardiomyocytes beat best on a matrix with heart-like elasticity: scar-like rigidity inhibits beating. J Cell Sci 121: 3794-3802.

Engler, A.J., M.A. Griffin, S. Sen, C.G. Bönnemann, H.L. Sweeney, D.E. Discher (2004) Myotubes differentiate optimally on substrates with tissue-like stiffness: pathological implications for soft or stiff microenvironments. J Cell Biol 166: 877-887.

Fernandes, S., J.C. Amirault, G. Lande, J.M. Nguyen, V. Forest, O. Bignolais, G. Lamirault, D. Heudes, J.L. Orsonneau, M.F. Heymann, F. Charpentier, P. Lemarchand (2006) Autologous myoblast transplantation after myocardial infarction increases the inducibility of ventricular arrhythmias. Cardiovasc Res 69: 348-358.
Forest, V.F., A.M. Tirouvanziam, C. Perigaud, S. Fernandes, M.S. Fusellier, J.C. Desfontis, C.S. Toquet, M.F. Heymann, D.P. Crochet, P.F. Lemarchand (2010) Cell distribution after intracoronary bone marrow stem cell delivery in damaged and undamaged myocardium: implications for clinical trials. Stem Cell Res Ther 1: 4

-García, A.J., M.D. Vega, D. Boettiger (1999) Modulation of cell proliferation and differentiation through substrate-dependent changes in fibronectin conformation. Mol Biol Cell 10: 785-798.

Gilbert, P.M., K.L. Havenstrite, K.E. Magnusson, A. Sacco, N.A. Leonardi, P. Kraft, N.K. Nguyen, S. Thrun, M.P. Lutolf, H.M. Blau (2010) Substrate elasticity regulates skeletal muscle stem cell self-renewal in culture. Science 329: 1078-1081.

Jacot, J.G., H. Kita-Matsuo, K.A. Wei, H.S. Chen, J.H. Omens, M. Mercola, A.D. McCulloch (2010) Cardiac myocyte force development during differentiation and maturation. Ann NY Acad Sci 1188: 121-127.

Jacot, J.G., A.D. McCulloch, J.H. Omens (2008) Substrate stiffness affects the functional maturation of neonatal rat ventricular myocytes. Biophys J 95: 3479-3487.

Jain, M., H. DerSimonian, D.A. Brenner, S. Ngoy, P. Teller, A.S. Edge, A. Zawadzka, K. Wetzel, D.B. Sawyer, W.S. Colucci, C.S. Apstein, R. Liao (2001) Cell therapy attenuates deleterious ventricular remodeling and improves cardiac performance after myocardial infarction. Circulation 103: 1920-1927.

Kocher, A.A., M.D. Schuster, M.J. Szabolcs, S. Takuma, D. Burkhoff, J. Wang, S. Homma, N.M. Edwards, S. Itescu (2001) Neovascularization of ischemic myocardium by human bone-marrow-derived angioblasts prevents cardiomyocyte apoptosis, reduces remodeling and improves cardiac function. Nat Med 7: 430-436.

Kofidis, T., D.R. Lebl, E.C. Martinez, G. Hoyt, M. Tanaka, R.C. Robbins (2005) Novel injectable bioartificial tissue facilitates targeted, less invasive, large-scale tissue restoration on the beating heart after myocardial injury. Circulation 112(9 suppl): I173-177. 
Kraehenbuehl, T.P., L.S. Ferreira, A.M. Hayward, M. Nahrendorf, A.J. van der Vlies, E. Vasile, R. Weissleder, R. Langer, J.A. Hubbell (2011) Human embryonic stem cell-derived microvascular grafts for cardiac tissue preservation after myocardial infarction. Biomaterials 32: 1102-1109.

Kääriäinen, M., T. Järvinen, M. Järvinen, J. Rantanen, H. Kalimo (2000) Relation between myofibers and connective tissue during muscle injury repair. Scand J Med Sci Sports 10: 332-337.

Laflamme, M.A., K.Y. Chen, A.V. Naumova, V. Muskheli, J.A. Fugate, S.K. Dupras, H. Reinecke, C. Xu, M. Hassanipour, S. Police, C. O’Sullivan, L. Collins, Y. Chen, E. Minami, E.A. Gill, S. Ueno, C. Yuan, J. Gold, C.E. Murry (2007a) Cardiomyocytes derived from human embryonic stem cells in prosurvival factors enhance function of infarcted rat hearts. Nat Biotechnol 25: 1015-1024.

Laflamme, M.A., C.E. Murry (2011) Heart regeneration. Nature 473: 326-335.

Laflamme, M.A., S. Zbinden, S.E. Epstein, C.E. Murry (2007b) Cell-based therapy for myocardial ischemia and infarction: pathophysiological mechanisms. Annu Rev Pathol 2: 307-339.

LaNasa, S.M., S.J. Bryant (2009) Influence of ECM proteins and their analogs on cells cultured on 2-D hydrogels for cardiac muscle tissue engineering. Acta Biomater 5: 29292938.

Leobon, B., I. Garcin, P. Menasche, J.T. Vilquin, E. Audinat, S. Charpak (2003) Myoblasts transplanted into rat infarcted myocardium are functionally isolated from their host. Proc Natl Acad Sci USA 100: 7808-7811.

-Leor, J., Y. Amsalem, S. Cohen (2005) Cells, scaffolds, and molecules for myocardial tissue engineering. Pharmacol Ther 105: 151-163.

Leor, J., S. Gerecht, S. Cohen, L. Miller, R. Holbova, A. Ziskind, M. Shachar, M.S. Feinberg, E. Guetta, J. Itskovitz-Eldor (2007) Human embryonic stem cell transplantation to repair the infarcted myocardium. Heart 93: $1278-1284$

Leor, J., M. Patterson, M.J. Quinones, L.H. Kedes, R.A. Kloner (1996) Transplantation of fetal myocardial tissue into the infarcted myocardium of rat: a potential method for repair of infarcted myocardium? Circulation 94(suppl): II332-336.

Levy-Mishali, M., J. Zoldan, S. Levenberg (2009) Effect of scaffold stiffness on myoblast differentiation. Tissue Eng Part A 15: 935-944.

Moon, J.J., J.L. West (2008) Vascularization of engineered tissues: approaches to promote angio-genesis in biomaterials. Curr Top Med Chem 8:300-310.
Murry, C.E., H. Reinecke, L.M. Pabon (2006) Regeneration gaps: observations on stem cells and cardiac repair. J Am Coll Cardiol 47: 1777-1785.

Murry, C.E., R.W. Wiseman, S.M. Schwartz, S.D. Hauschka (1996) Skeletal myoblast transplantation for repair of myocardial necrosis. J Clin Invest 98: 2512-2523.

Müller-Ehmsen, J., K.L. Peterson, L. Kedes, P. Whittaker, J.S. Dow, T.I. Long, P.W. Laird, R.A. Kloner (2002) Rebuilding a damaged heart: long-term survival of transplanted neonatal rat cardiomyocytes after myocardial infarction and effect on cardiac function. Circulation 105: 1720-1726.

Nelson, D.M., Z. Ma, K.L. Fujimoto, R. Hashizume, W.R. Wagner (2011) Intra-myocardial biomaterial injection therapy in the treatment of heart failure: materials, outcomes and challenges. Acta Biomater 7: 1-15.

Nygren, J.M., S. Jovinge, M. Breitbach, P. Säwén, W. Röll, J. Hescheler, J. Taneera, B.K. Fleischmann, S.E. Jacobsen (2004) Bone marrowderived hematopoietic cells generate cardiomyocytes at a low frequency through cell fusion, but not transdifferentiation. Nat Med 10: 494-501.

-Ono, Y., H. Sensui, Y. Sakamoto, R. Nagatomi (2006) Knockdown of hypoxia-inducible factor-1alpha by siRNA inhibits C2C12 myoblast differentiation. J Cell Biochem 98: 642649.

Padin-Iruegas, M.E., Y. Misao, M.E. Davis, V.F. Segers, G. Esposito, T. Tokunou, K. Urbanek, T. Hosoda, M. Rota, P. Anversa, A. Leri, R.T. Lee, J. Kajstura (2009) Cardiac progenitor cells and biotinylated insulin-like growth factor-1 nanofibers improve endogenous and exogenous myocardial regeneration af ter infarction. Circulation 120: 876-887.

Pouzet, B., S. Ghostine, J.T. Vilquin, I. Garcin, M. Scorsin, A.A. Hagège, D. Duboc, K. Schwartz, P. Menasché (2001) Is skeletal myoblast transplantation clinically relevant in the era of angiotensin-converting enzyme inhibitors? Circulation 104(suppl 1): I223-228.

Reinecke, H., G.H. MacDonald, S.D. Hauschka, C.E. Murry (2000) Electromechanical coupling between skeletal and cardiac muscle. Implications for infarct repair. J Cell Biol 149: 731-740.

Robey, T.E., C.E. Murry (2008) Absence of regeneration in the MRL/MpJ mouse heart following infarction or cryoinjury. Cardiovasc Pathol 17: 6-13.

Robey, T.E., M.K. Saiget, H. Reinecke, C.E. Murry (2008) Systems approaches to preventing transplanted cell death in cardiac repair. J Mol Cell Cardiol 45: 567-581.
Schechner, J.S., A.K. Nath, L. Zheng, M.S. Kluger, C.C. Hughes, M.R. Sierra-Honigmann, M.I. Lorber, G. Tellides, M. Kashgarian, A.L. Bothwell, J.S. Pober (2000) In vivo formation of complex microvessels lined by human endothelial cells in an immunodeficient mouse. Proc Natl Acad Sci USA 97: 9191-9196.

Scorsin, M., A. Hagège, J.T. Vilquin, M. Fiszman, F. Marotte, J.L. Samuel, L. Rappaport, K. Schwartz, P. Menasché (2000) Comparison of the effects of fetal cardiomyocyte and skeletal myoblast transplantation on postinfarction left ventricular function. J Thorac Cardiovasc Surg 119: 1169-1175.

Serrano, A.L., P. Muñoz-Cánoves (2010) Regulation and dysregulation of fibrosis in skeletal muscle. Exp Cell Res 316: 3050-3058.

Tang, X.L., G. Rokosh, S.K. Sanganalmath, F. Yuan, H. Sato, J. Mu, S. Dai, C. Li, N. Chen, Y. Peng, B. Dawn, G. Hunt, A. Leri, J. Kajstura, S. Tiwari, G. Shirk, P. Anversa, R. Bolli (2010) Intracoronary administration of cardiac progenitor cells alleviates left ventricular dysfunction in rats with a 30-day-old infarction. Circulation 121: 293-305.

van Laake, L.W., E.G. van Donselaar, J. Monshouwer-Kloots, C. Schreurs, R. Passier, B.M. Humbel, P.A. Doevendans, A. Sonnenberg, A.J. Verkleij, C.L. Mummery (2010) Extracellular matrix formation after transplantation of human embryonic stem cell-derived cardiomyocytes. Cell Mol Life Sci 67: 277-290.

Wall, S.T., C.C. Yeh, R.Y. Tu, M.J. Mann, K.E. Healy (2010) Biomimetic matrices for myocardial stabilization and stem cell transplantation. J Biomed Mater Res A 95: 1055-1066.

-Yasuda, T., R.D. Weisel, C. Kiani, D.A. Mickle, M. Maganti, R.K. Li (2005a) Quantitative analysis of survival of transplanted smooth muscle cells with real-time polymerase chain reaction. J Thorac Cardiovasc Surg 129: 904-911.

Yasuda, T., K. Yasuda, R.A. Brown, J.M. Krueger (2005b) State-dependent effects of light-dark cycle on somatosensory and visual cortex EEG in rats. Am J Physiol Regul Integr Comp Physiol 289: R1083-1089.

Yu, J., K.L. Christman, E. Chin, R.E. Sievers, M. Saeed, R.J. Lee (2009) Restoration of left ventricular geometry and improvement of left ventricular function in a rodent model of chronic ischemic cardiomyopathy. J Thorac Cardiovasc Surg 137: 180-187.

Zhang, M., D. Methot, V. Poppa, Y. Fujio, K. Walsh, C.E. Murry (2001) Cardiomyocyte grafting for cardiac repair: graft cell death and anti-death strategies. J Mol Cell Cardiol 33: 907-921. 